\title{
ROS-inducing potential, influence of different porogens and in vitro degradation of poly (D,L-lactide-co-glycolide)-based material
}

\author{
M. Stevanović ${ }^{*}$, V. Pavlović ${ }^{2}$, J. Petković3 ${ }^{3}$, M. Filipič ${ }^{3}$, D. Uskoković ${ }^{1}$ \\ ${ }^{1}$ Institute of Technical Sciences of the Serbian Academy of Sciences and Arts, Knez Mihailova 35/IV, Belgrade 11000, \\ Serbia \\ ${ }^{2}$ Faculty of Agriculture, University of Belgrade, Nemanjina 6, Belgrade 11080, Serbia \\ ${ }^{3}$ Department of Genetic Toxicology and Cancer Biology, National Institute of Biology, Vecna pot 111, Ljubljana 1000, \\ Slovenia
}

Received 18 March 2011; accepted in revised form 2 June 2011

\begin{abstract}
Porous, poly(D,L-lactide-co-glycolide) (PLGA) materials were prepared by physicochemical solvent/non-solvent method with polyvinyl pyrrolidone (PVP) as a stabilizer and with silicone oil, paraffin, hydrogen peroxide or sodium chloride as a porogen. The obtained PLGA particles without porogens are non-agglomerated, uniform and with particle size on the submicron scale. The formation of intracellular reactive oxygen species (ROS) was measured spectrophotometrically using a fluorescent probe, 2,7-dichlorofluorescein diacetate (DCFH-DA) and it is shown that PLGA nanospheres are not inducers of intracellular formation. Porous PLGA scaffolds obtained in the experiment with sodium chloride as porogen and water as solvent of the porogen had apparently uniform pore morphology with spheroidal pore in shape and well controlled three-dimensional interconnected network. PLGA scaffolds are highly porous with similar porosity values. The degradation of PLGA nanoparticles and PLGA porous materials were studied in phosphate buffered saline as a degradation medium. The samples were characterized by Infrared Spectroscopy (IR), X-ray difractometry, Zeta potential measurements, Scanning Electron Microscopy (SEM) and Ultraviolet Spectroscopy (UV).
\end{abstract}

Keywords: biodegradable polymers, ROS formation, porogens, zeta potential, in vitro degradation

\section{Introduction}

A variety of materials have been used for replacement and repair of damaged or traumatized tissues [1-8].These materials include metals, ceramics, polymers (natural and synthetic) and their combinations. Polymers have great design flexibility because their composition and structure can be tailored to the specific needs $[9,10]$. They are therefore attractive candidates for various clinical applications. Synthetic biodegradable polymers, poly (esters) based on polylactide (PLA), polyglycolide (PGA), polycaprolactone (PCL) and their copolymers are approved by the World Health Organization (WHO) and Food and Drug Administration (FDA) as materials that can be used in medicine and pharmacy [11-14]. Poly (DL-lactide-co-glycolide) (PLGA), a biocompatible and biodegradable copolymer of PLA and PGA, is widely used in various medical applications; controlled release of delivering drugs, scaffolds in the tissue engineering, fixation of bone fractures, chirurgical strings, etc. [15-17]. PLGA particles are used for the controlled delivery of several types of medicaments, including anticancer agents, antihypertensive agents, immunomodulatory drugs, hormones, vitamins, etc. [18-20].

\footnotetext{
${ }^{*}$ Corresponding author, e-mail: magdalena.stevanovic@itn.sanu.ac.rs
} (c) BME-PT 
Scaffold provides temporary support for cell proliferation and differentiated function, allowing neotissue formation and initial remodeling [19]. The choice of the best scaffold for a particular tissue engineering application and how to change an existing scaffold to improve its performance are important components of any tissue engineering research project [19]. Numerous techniques have been used for scaffold design, such as solvent casting/particulate leaching, phase separation, microsphere sintering, electrospining, emulsion freeze drying, fiber extrusion, gas foaming and 3-D printing [19-21].

Many factors are important when designing materials for use in biomedicine. The main advantage of nano and microspheres used in tissue engineering, compared to the more traditional macroporous block scaffolds, is that nano and microspheres possess not only better drug-delivery properties, but also the ability to fill the bone defects with irregular and complex shapes and sizes [22]. The interstitial space between the particles of the microspheres is crucial for effective and functional bone regeneration [22-24] as they allow for both bone and vascular ingrowths. Appropriate porosity is a crucial biomaterial design criterion for materials used in tissue engineering applications as it can allow increased cell adhesion, migration, proliferation and extracellular matrix production within the scaffold at a tissue defect site [25].

In controlled drug delivery porosity is also a very important factor influencing the degradation process of poly(DL-lactide-co-glycolide). The presence of pores does not only increase the mobility of the involved species (drug molecules, acids and bases), but fundamentally alters the underlying drug release mechanisms. Generally, the release of medicament during the degradation process will be faster in case of porous particles. A factor related to the sphere porosity is, also, the initial burst effect, which corresponds to the rapid initial release of drug and is normally followed by the relatively controlled linear release [26].

The goal of the study reported here was to investigate the influence of different porogens on the morphological characteristics and formation of pores in PLGA based materials, nano and microspheres obtained by physicochemical solvent/non-solvent method, and also very important to eliminate the release-delayed phenomenon of macromolecular drug delivery system at the early stage of the degradation. Porous, PLGA materials were prepared with polyvinyl pyrrolidone (PVP) as a stabilizer and with silicone oil, paraffin, hydrogen peroxide or sodium chloride as a porogen. PVP acting as stabilizer and dispersant i.e. has role to prevent coalescence or aggregation of the particles. In the process of the preparation of the porous PLGA, we have obtained non-particulate templates in the forms of porous films and scaffolds. Using the synthesis protocol nanoparticulate PLGA materials were obtained only in the absence of porogens [27]. Since the recent studies have pointed out that the higher toxic potential of nanomaterials in comparison to their larger counterparts is most probably due to oxidative stress as a consequence of increased production of reactive oxygen species (ROS) [28, 29], it is very important to establish ROS-inducing potential of our model nanomaterial, PLGA nanospheres, which is determined in this paper.

\section{Experimental}

\subsection{Materials}

Poly(DL-lactide-co-glycolide) (PLGA) was purchased from Durect, Lactel (Birmingham, Alabama, USA) and had a lactide-to-glycolide ratio of 50:50. Molecular weight of the polymer is 40000 $50000 \mathrm{~g} / \mathrm{mol}$. The time for its complete resorption in the body is 4 to 8 weeks. Polyvinyl pyrrolidone (povidone, PVP) was obtained from Merck Chemicals Ltd (k-25, Merck, Germany). Silicon oil, sodium chloride, hydrogen peroxide (all from Centrohem, Serbia) and paraffin (melting point $53-57^{\circ} \mathrm{C}$, Merck, Germany) were used as porogens. Cyclohexane (boiling point $81{ }^{\circ} \mathrm{C}$, Merck, Germany), n-heptane (Centrohem, Serbia) and distilled water were used as solvents of a porogens.

The degradation medium used was phosphate buffered saline (PBS, one tablet dissolved in $200 \mathrm{ml}$ of deionized water yields $0.137 \mathrm{~mol} / \mathrm{l}$ sodium chloride, $0.01 \mathrm{~mol} / 1$ phosphate buffer and $0.0027 \mathrm{~mol} / 1$ potassium chloride, $\mathrm{pH}$ is 7.4 at $25^{\circ} \mathrm{C}$, SigmaAldrich, St. Louis, USA) with sodium azide (SigmaAldrich Chemie GmbH Germany ), $0.1 \mathrm{~mol} / 1$ solution $\mathrm{NaN}_{3}$ ).

Chemicals for determining intracellular reactive oxygen species formation (DCFH-DA assay) [30], Minimal Essential Medium Eagle, penicillin/streptomycin, L-glutamine, phosphate buffered saline, 
trypsin, foetal bovine serum, non-essential amino acid solution $(100 \times)$, and 2,7-dichlorofluorescein diacetate (DCFH-DA) were obtained from Sigma Aldrich (St. Louis, USA).

\subsection{Preparation of PLGA nanospheres and preparation of porous PLGA samples by different porogens and different solvents of the porogens}

PLGA nano and microparticles were synthesized following method previously described in our work [31] i.e. by using physicochemical method with solvent/nonsolvent systems where obtained solutions were centrifuged. Polyvinyl pyrrolidone was used as a stabilizer of the particles.

Briefly, commercial granules poly(D,L-lactide-coglycolide) $(250 \mathrm{mg})$ have been dissolved in the acetone $(20 \mathrm{ml})$ and, after approximately two hours, ethanol $(25 \mathrm{ml})$ has been added into the solvent mixture. PLGA was precipitated by the addition of ethanol and the solution became whitish. The polymeric solution thus obtained has been very slowly poured into aqueous PVP solution $(0.05 \% \mathrm{w} / \mathrm{w})$ while continuously stirring at $1200 \mathrm{rpm}$ by a stirrer. After that porogen (silicon oil, paraffin, hydrogen peroxide or sodium chloride) has been added into the mixture and then the solution has been centrifuged at $6000 \mathrm{rpm}$ and at $5^{\circ} \mathrm{C}$ for $120 \mathrm{~min}$ (Universal 320R, Hettich, Germany) and decanted. In all experiments PLGA:porogen ratio was 1:10. This ratio was used because; in case porogen content is sufficiently high, the resulting pores must be with some interconnectivity simply due to geometrical packing. The sediment was placed into the Petri dish and allowed to dry overnight at room temperature. The dried polymer films were removed from the Petri dish and soaked in appropriate solvent for $72 \mathrm{~h}$ to remove the porogen. The solvent was changed, approximately, every $12 \mathrm{~h}$ to allow the porogen to leach out. Paraffin as porogen has been removed from polymeric film with cyclohexane, silicone oil with n-heptan and sodium chloride with distilled water. This was repeated several times to remove the porogen in its entirety. PLGA films were vacuum dried (Vaciotem P-Selecta) for $48 \mathrm{~h}$ to allow the solvent to evaporate.

\subsection{The structural analysis of the samples}

In order to investigate the characteristics of the PLGA particles and PLGA porous materials obtained with different porogens i.e. to confirm the quality composition of the samples, X-ray diffraction (XRD) has been used. Phase analyses were performed by XRD, using a Philips PW 1050 diffractometer with $\mathrm{Cu}-\mathrm{K}_{\alpha 1,2}$ radiation (Ni filter). Measurements were done in $2 \theta$ range of $10-70^{\circ}$ with scanning step width of $0.05^{\circ}$ and $2 \mathrm{~s}$ of time per step.

The quality analysis of the samples was, also, performed using IR spectroscopy. IR spectra were recorded in the range of $400-4000 \mathrm{~cm}^{-1}$ at a MIDAC M 2000 Series Research Laboratory FTIR Spectrometer, at $4 \mathrm{~cm}^{-1}$ resolution.

\subsection{Zeta potential measurements}

Zeta potential was measured by Zetasizer (Nano ZS, Model ZEN3600, particles size range for zeta potential determination $(5 \mathrm{~nm}-10 \mu \mathrm{m})$, Malvern Instruments, Malvern, UK) using the principle of electrophoretic mobility under an electric field. Zeta potential is the function of dispersion/suspension $\mathrm{pH}$ which determines particle stability in dispersion.

\subsection{Determining intracellular reactive oxygen species formation - DCFH-DA assay}

The formation of intracellular reactive oxygen species (ROS) was measured spectrophotometrically using a fluorescent probe, DCFH-DA as described by Osseni et al. [30], with minor modifications [32]. DCFH-DA readily diffuses through the cell membrane and is hydrolyzed by intracellular esterases to non-fluorescent 2',7'-dichlorofluorescin. It is then rapidly oxidized to highly fluorescent $2^{\prime}, 7^{\prime}$-dichlorofluorescein in the presence of ROS. The DCF fluorescence intensity is proportional to the amount of reactive oxygen species formed intracellularly. $\mathrm{H}_{2} \mathrm{O}_{2}$ is the principle ROS responsible for the oxidation of DCFH-DA to DCF [33].

In this assay we used cells HepG2, which were grown in Minimal Essential Medium Eagle medium containing $10 \%$ foetal bovine serum, $1 \%$ non-essen- 
tial amino acid solution, $2 \mathrm{mM}$ L-glutamine and $100 \mathrm{U} / \mathrm{ml}$ penicillin plus $100 \mu \mathrm{g} / \mathrm{ml}$ streptomycin at $37^{\circ} \mathrm{C}$ in humidified atmosphere and $5 \% \mathrm{CO}_{2}$. HepG2 cells were seeded at a density of 75000 cells/ml into 96-well, black, tissue culture treated microtiter plates (Nunc, Naperville IL, USA) in five replicates. After $20 \mathrm{~h}$ of incubation at $37^{\circ} \mathrm{C}$ in $5 \% \mathrm{CO}_{2}$, cells were incubated with $20 \mu \mathrm{M}$ DCFH-DA. After $30 \mathrm{~min}$, DCFH-DA was removed and cells were treated with $0,1,10,25,50$ and $66,5 \mu \mathrm{g} / \mathrm{ml}$ of PLGA nanoparticles without porogens in PBS. In each experiment were included negative control (non-treated cells), positive control $(0,5 \mathrm{mM}$ t-BOOH) and a vehicle control ( $5 \%$ emulsion; emulsion consists of acetone, ethanol and PVP, in the same ratio as used in the experiment, without PLGA) in order to exclude possible effects of the solvent (emulsion). For kinetic analyses the dishes were maintained at $37^{\circ} \mathrm{C}$ and the fluorescence intensity was determined every $30 \mathrm{~min}$ during the $5 \mathrm{~h}$ incubation using a microplate reading spectrofluorimeter (Tecan, Genios) at the excitation wavelength of $485 \mathrm{~nm}$ and the emission wavelength of $530 \mathrm{~nm}$.

Statistical significance between treated groups and controls was determined by two tailed Student's ttest and $P<0.05$ was considered as statistically significant. Two independent experiments with five replicates were performed.

In our study we tested ROS-inducing potential of PLGA nanoparticles without porogens only and we did not test ROS-inducing potential of porous PLGA material, since it is much more difficult to perform testing of material in form of the scaffold than of particles that can be dispersed. Testing of materials in the form of scaffold requires seeding of cells onto material surface instead of seeding onto plastic surfaces, which is regularly used for in vitro toxicology tests. For the analysis of ROS-inducing potential of such material it is necessary to detach the cells from the material (with trypsinization or cell scraping), process that itself generates cellular oxidative stress and result in artificial changes in fluorescence, due to which we can observe false positive results. Because of this we tested only ROS-inducing potential of PLGA nanoparticles without porogens.

\subsection{Morphology studies}

The morphology of obtained nano and microparticles and porous samples was evaluated by scanning electron microscope (SEM) (JEOL JSM-639OLV) and SUPRA 35 VP Carl Zeiss field emission scanning electron microscope (FESEM). The powder samples for SEM analysis were coated with gold using the physical vapour deposition (PVD) process. Samples were covered with gold (Baltec SCD 005 sputter coater), using $30 \mathrm{~mA}$ current from the distance of $50 \mathrm{~mm}$ during $180 \mathrm{~s}$. SEM recordings were, also, used to obtain information about profiles and sizes of the particles and pores.

\subsection{Water absorption}

Water absorption of the polymeric films or scaffolds was measured in the manner described in detail elsewhere [34-38]. Water absorption was measured by immersing the samples in distilled water for a predetermined time span (55 hours), then the samples were taken out and dried by removing the free water on the surface with filter paper and weighed (W1). Then the samples were thoroughly vacuum-dried and weighed again (W2). The water absorption could be calculated as shown in Equation (1) and five specimens were averaged.

Water absorption $[\%]=\frac{\mathrm{W} 1-\mathrm{W} 2}{\mathrm{~W} 2} \cdot 100$

\subsection{In vitro degradation studies}

The degradation of PLGA nanoparticles and PLGA porous materials obtained with sodium chloride as porogen were studied during the 39 days in phosphate buffered saline as a degradation medium.

Taking into account that the degradation products of PLGA, among others, are lactic and glycolic acid [31], a calibration curve for lactic acid was made. By tracking the increase of the concentration of lactic acid in the solution, the degradation of the samples was monitored.

The concentration of lactic acid in the phosphate buffered saline as a release medium was determined by UV spectroscopy. Based on measuring absorbance of the solution with a known concentration of lactic acid at $220 \mathrm{~nm}$, a calibration curve was prepared. The linear relationship between light absorbance at 
$220 \mathrm{~nm}$ and lactic acid concentration is shown according to the Beer-Lambert Law (Equation (2)):

$A=\varepsilon c l$

where $A$ is absorbance at sample concentration $c$ (in this case concentration of the lactic acid $[\mathrm{mg} / \mathrm{ml}]$ ), $l$ is path length of quartz cell and $\varepsilon$ is the absorptivity. By applying this standardized relationship, PBS solution, in which the samples were suspended, was analyzed to determine the concentration and amount of lactic acid as product of the degradation of PLGA samples whether it is porous or whether it is not porous. The in vitro degradation of PLGA nanoparticles and PLGA porous materials obtained with sodium chloride as porogen was studied by suspending samples in PBS in the absence of light at $37 \pm 1^{\circ} \mathrm{C}$ (VIMS elektrik, SCG). Measurements of the degradation of both samples were undertaken by exposing $2 \mathrm{mg}$ of the sample to $2 \mathrm{ml}$ of PBS. The UV measurements were performed on GBC, Cintra 101 UV-VIS Spectrophotometer in the frequency interval of 200-400 nm.

\section{9. pH measurements}

The $\mathrm{pH}$ of the physiological solution has been measured using $\mathrm{pH}$ indicator strips obtained from Merck (KGaA, Germany) at various time periods (during the 39 days) to follow the acidity of the degrading medium with time.

\section{Results and discussion}

\subsection{The structural analysis of the samples}

The IR spectra illustrate all characteristic groups for copolymer poly(DL-lactide-coglycolide). The IR spectra of PLGA show peaks at 2995, 2948 , $2841 \mathrm{~cm}^{-1}\left(\mathrm{C}-\mathrm{H}\right.$ bend of the $-\mathrm{CH}_{2}$ and $-\mathrm{CH}_{3}$ group), $1769 \mathrm{~cm}^{-1}(\mathrm{C}=\mathrm{O}$ ester $), 1461,1425$, $1370 \mathrm{~cm}^{-1}$ (C-H bend of the $\mathrm{CH}_{3}$ group), 1150 , $1069,987 \mathrm{~cm}^{-1}$ (C-O stretch), 735, $510 \mathrm{~cm}^{-1}$ (C-H bend). The IR spectra, also, shows a broad band in the range $3100-3600 \mathrm{~cm}^{-1}$ which belongs to the $\mathrm{OH}-$ group of the water molecule $[39,40]$.

The X-ray diffractograms of PLGA nanoparticles and PLGA porous materials are presented in Figure 1. PLGA did not exhibit any crystalline peak

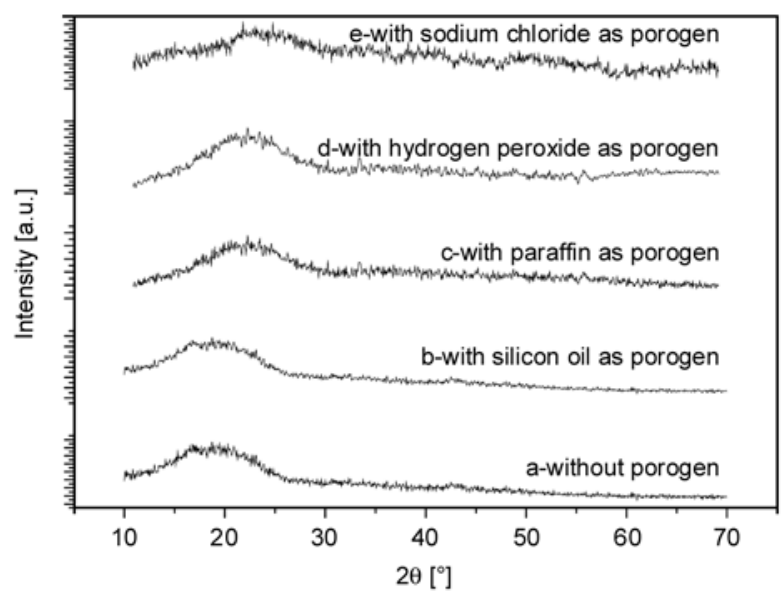

Figure 1. XRD patterns of a) PLGA particles prepared without porogen, b) PLGA obtained with silicone oil as porogen and n-heptan as solvent of the porogen; c) PLGA obtained with paraffin as porogen and cyclohexane as solvent of the porogen; d) PLGA obtained with hydrogen peroxide and e) PLGA obtained with sodium chloride as porogen and water as solvent of the porogen

under the present experimental conditions because PLGA is amorphous. Also, X-ray patterns show that there is no presence of residual porogens in the samples (Figure 1b-d). On the Figure 1e strong background and the up-shift of the broad structure can be observed. We may assume this is probably related to residual sodium chloride. However, as known from the literature [41], sodium chloride is crystalline and has strong peaks at $2 \theta=31.8,45.6$ and $66.2^{\circ}$ assigned to (200), (400) and (220) planes respectively, which are not present. Thus we conclude the amount of the residual sodium chloride is negligible.

\subsection{Zeta potential measurements}

PVP was used as a stabilizer which creates negatively charged PLGA particles, that is, induces a specific zeta potential. PVP reduces the agglomeration because the particles of the same charge are not attracted to each other. A refractive index (RI) value of 1.4 was used in determination of the size of nanoparticles. The RI value is estimated from product literature values for the RI of polylactic acid (1.35-1.45, Natureworks LLC) and for the monomers (lactic acid $=1.42$, glycolic acid $=1.41$, Sigma-

Table 1. Zeta potential of PLGA dispersion and size of PLGA particles in dispersion

\begin{tabular}{|c|c|c|c|c|}
\hline & Particle size [nm] & pH & Polydispersity index & Zeta potential [mV] \\
\hline PLGA2 & $275 \pm 5$ & $4.30-4.37$ & 0.053 & $-9.5 \pm 0.3$ \\
\hline
\end{tabular}

Values given are mean \pm standard deviation $(n=5)$. 
Aldrich Corp.) [42]. The polydispersity index (PDI) which is a dimensionless number indicating the width of the size distribution, having a value between 0 and 1 ( 0 being for monodispersed particles) was also obtained (Table 1).

Values for the size of particles from the dispersion of the same sample determined by Malvern particle analyzer were bigger from the values obtained by SEM for particles of the powder of the same sample. The reason is, as we reported earlier [43], that time and velocity of centrifugal processing influences the morphology (size and shape) and uniformity of PLGA polymer powder. An influence of the centrifugation process on the particle sizes is supported by the fact that coefficient of friction for smaller and more compact spherical particles is less than those with irregular shapes and same mass, which makes spherical particles sediment faster [43]. Also, values for sizes of the particles in the dispersion can be bigger because of the hydrodynamic effect, layer of ions around the particles, aggregates of the particles, etc [44].

\subsection{Induction of intracellular ROS formation}

HepG2 cells are of human origin and retain many of the specialized liver functions and drug metabolizing enzyme activities present in human hepatocytes. They are considered to better reflect the processes in normal human liver than other in vitro

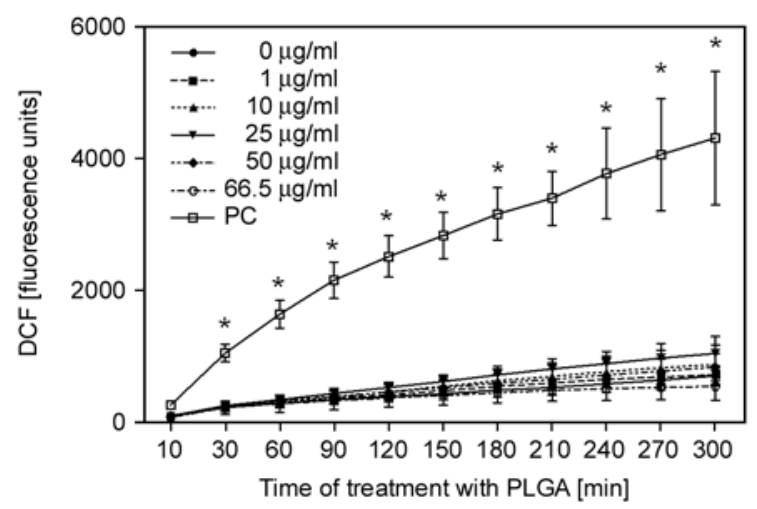

Figure 2. PLGA-induced intracellular ROS formation in HepG2 cells. The HepG2 cells were pretreated with DCFH-DA $(20 \mu \mathrm{M})$ for $30 \mathrm{~min}$, washed and then exposed to different concentrations of PLGA $(0,1,10,25,50$ and $66,5 \mu \mathrm{g} / \mathrm{ml})$ or $0,5 \mathrm{mM}$ t-BOOH as the positive control (PC). DCF fluorescence intensity was measured at $30 \mathrm{~min}$ intervals during the $5 \mathrm{~h}$ incubation. Each point represents the mean of five replicates $( \pm \mathrm{SD})$ of two independent experiments. (*) denotes a significant difference $(p<0,05)$. toxicity test systems. In our previous study we have shown that exposure of HepG2 cells to $0,1,10,25$, 50 and $66,5 \mu \mathrm{g} / \mathrm{ml}$ of PLGA nanoparticles for $24 \mathrm{~h}$ did not affect the viability of the cells [18]. Therefore, these concentrations were used in further experiments.

Exposure of HepG2 cells to PLGA nanoparticles did not induce significant increase of DCF fluorescence intensity, while exposure to $t$-BOOH, the positive control, induced significant, up to six fold increase in DCF fluorescence intensity over the control, non-treated cells (Figure 2). Based on these results we conclude that PLGA nanoparticles are not inducers of intracellular ROS formation.

\subsection{Morphology studies}

The morphological characteristics of the particles are extremely important for the controlled drug delivery and tissue engineering and particularly influence the adhesion and interaction with cells (intracellular uptake). Dynamics of the release (rate and concentration) depends on the morphology, i.e. structure of the copolymer.

The morphological characteristics of PLGA particles and PLGA porous materials obtained with different porogens, were observed by a scanning electron microscope. Different morphologies of PLGA were prepared using no porogen, and silicon oil, paraffin, hydrogen peroxide or sodium chloride as porogens. By using different porogens the samples with diferent morphological characteristics, at macroscopic and as well on microscopic level, were obtained.

From the micrograph of non-porous PLGA particles it is visible the particles have spherical shape, smooth surface, low level of agglomeration and high level of uniformity (Figure 3). The size distribution of all nanoparticles was unimodal with sizes of about $100-200 \mathrm{~nm}$. The particles are without cracks and pores.

From SEM images of PLGA material obtained in the experiment with silicone oil as porogen and n-hep$\tan$ as solvent of the porogen it is visible that use of silicon oil as porogen has no major impact on the formation of pores in the sample (Figure 4). The particles are much agglomerated and they create the film. On the film cracks and roughness can be seen. In the sample spherical particles of smaller and larger sizes that are distributed sporadically are still present. 

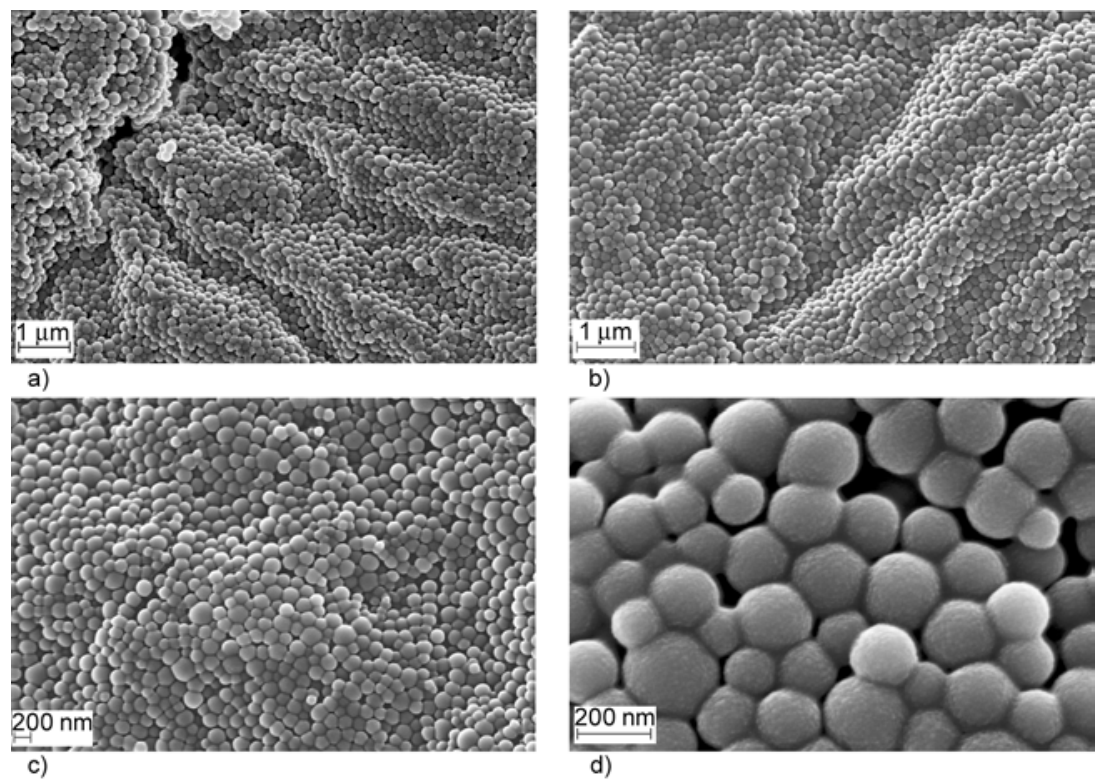

Figure 3. FESEM images of PLGA particles obtained in the experiment without porogen (a-d)

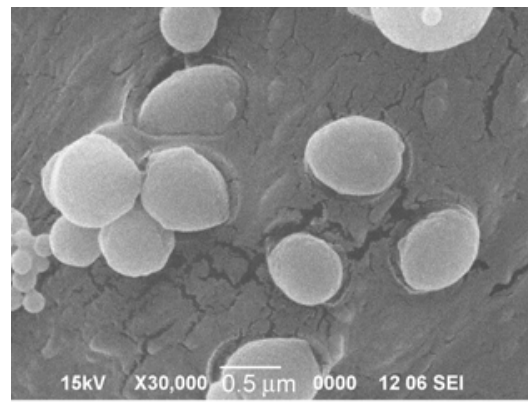

a)

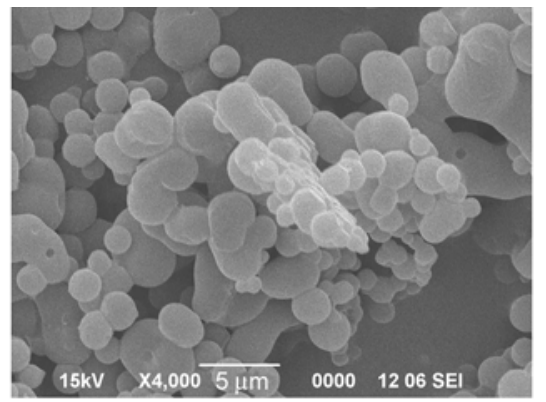

b)

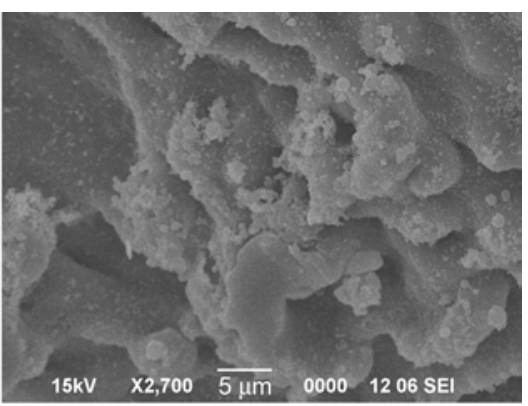

c)

Figure 4. SEM images of PLGA material obtained in the experiment with silicone oil as porogen and n-heptan as solvent of the porogen $(\mathrm{a}-\mathrm{c})$

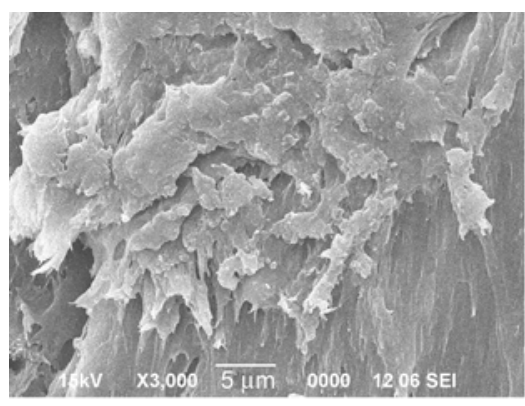

a)

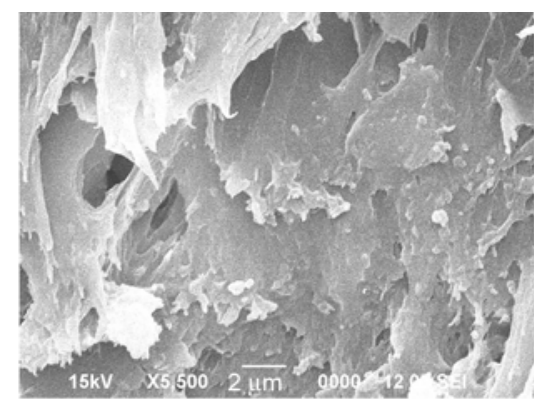

b)

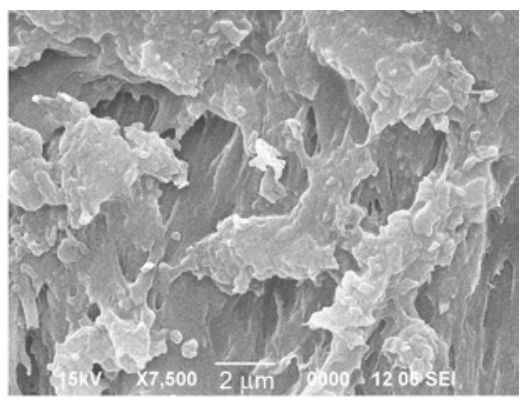

Figure 5. SEM images of PLGA material obtained in the experiment with paraffin as porogen and cyclohexane as solvent of the porogen $(\mathrm{a}-\mathrm{c})$

SEM images revealed porous morphologies of the samples when paraffin was used in the experiment as porogen and cyclohexane as solvent of the porogen (Figure 5). We can see from SEM micrographs that the resulting pores are with irregular shapes and without a good interconnectivity.
Figure 6 shows SEM observation of the interior pore structure of PLGA material obtained in the experiment when hydrogen peroxide was used as porogen. In this case the sample with high porosity and with good interconnectivity was obtained. The pores are with spherical but also irregular shapes. The pore size has mean value of about $10 \mu \mathrm{m}$. 


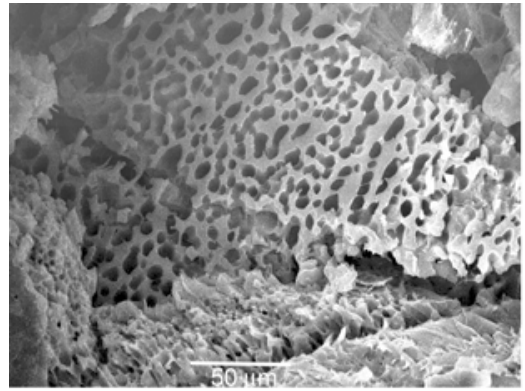

a)

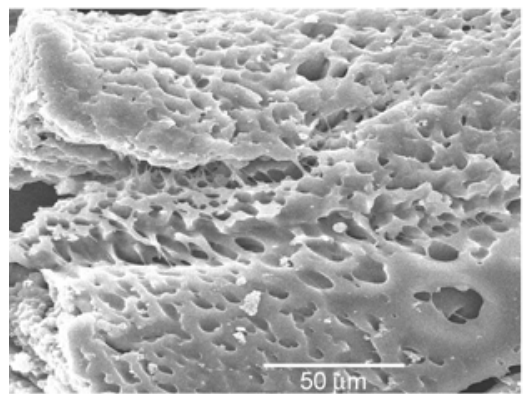

d)

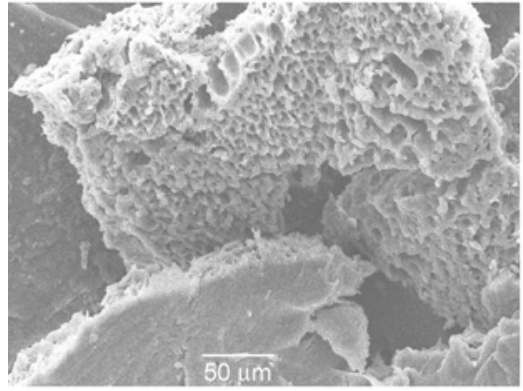

g)

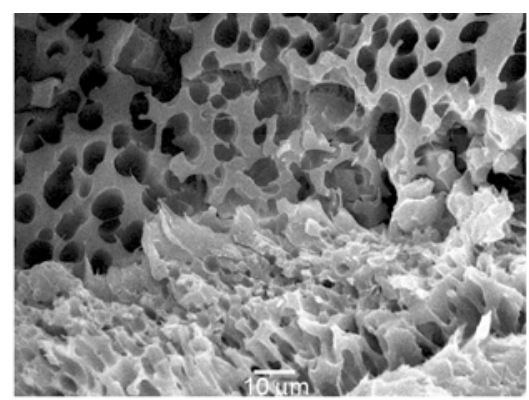

b)

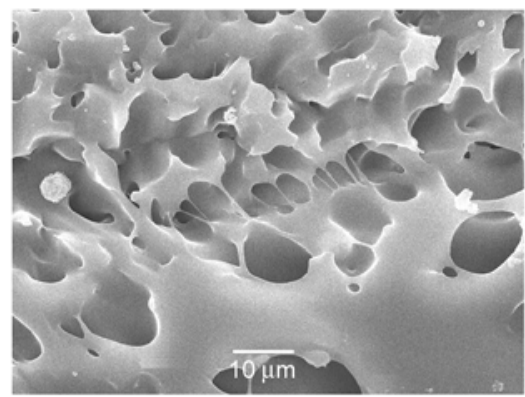

e)

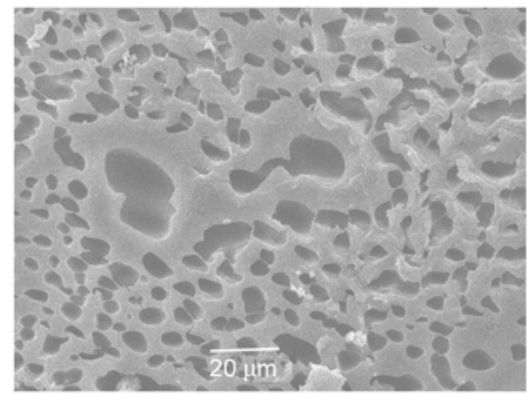

h)

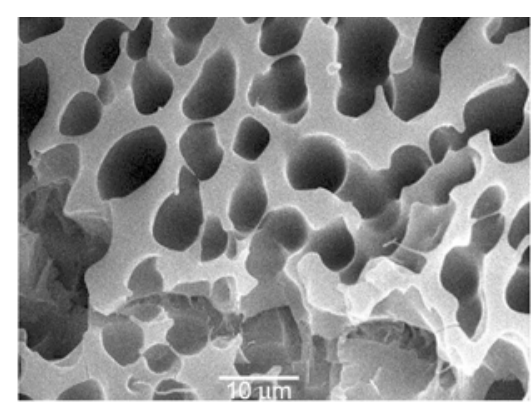

c)

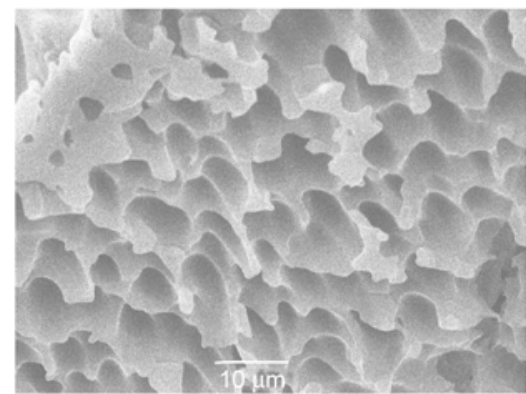

f)

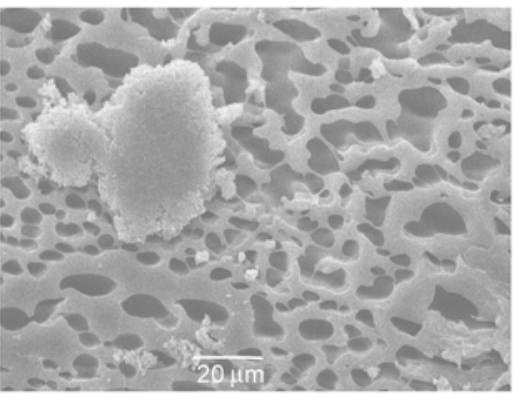

i)

Figure 6. SEM images of PLGA material obtained in the experiment with hydrogen peroxide as porogen (a-i)

Figure $7 \mathrm{a}-\mathrm{b}$ shows digital photographs of PLGA material obtained with sodium chloride as porogen. PLGA porous material appeared well formed and a highly porous sponge-like where the pores are similar in the size and shape.

The optimum morphological characteristics were obtained when sodium chloride was used as pore former. SEM micrographs showed that porous PLGA scaffolds obtained in the experiment with sodium chloride as porogen and water as solvent of the porogen had apparently uniform pore morphology with spheroidal pore in shape and well controlled three-dimensional interconnected network (Figure 7c-f). This PLGA samples are highly porous with similar porosity values. The pore size varies from 50 to $150 \mu \mathrm{m}$ based on SEM images. The unified and well organized porous structure can be seen. It is evident that pores have more precise contours.

\subsection{Water absorption}

In the literature, it has been reported that wetting of a polymer scaffold is very important for homogeneous and sufficient cell seeding throughout the porous scaffold [38]. To evaluate hydrophilicity of PLGA porous samples water absorption was measured. The water absorption of the samples changed with their porosity (Figure 8). PLGA porous scaffolds obtained with sodium chloride as porogen exhibited higher water absorption than other samples. It could be seen that the water absorption increased with the degree of porosity increasing and all the samples had higher water absorption than non-porous PLGA.

\subsection{Degradation studies}

The degradation of PLGA particles and PLGA porous materials obtained with sodium chloride as porogen was measured in PBS as a degradation 


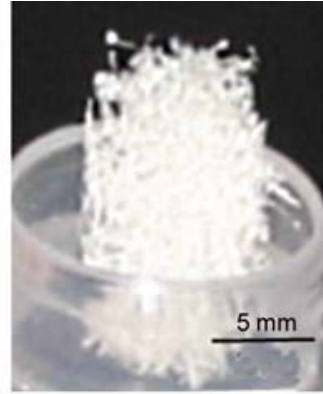

a)

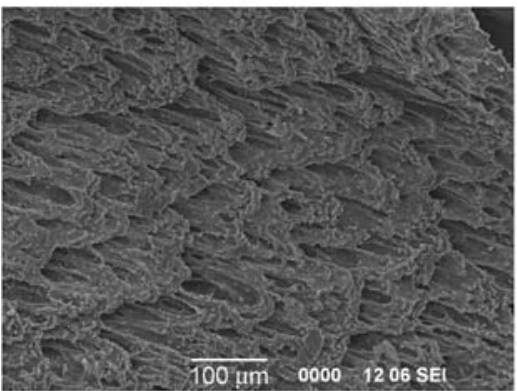

c)

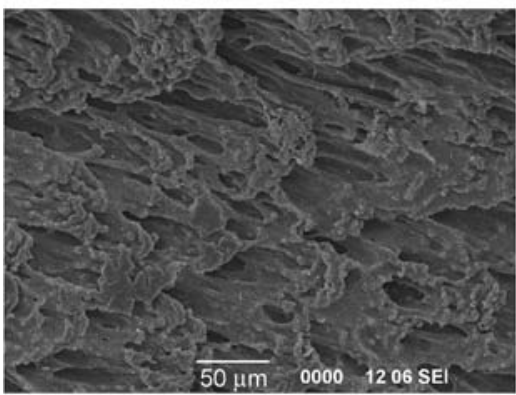

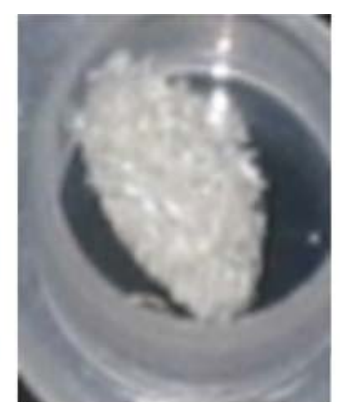

b)

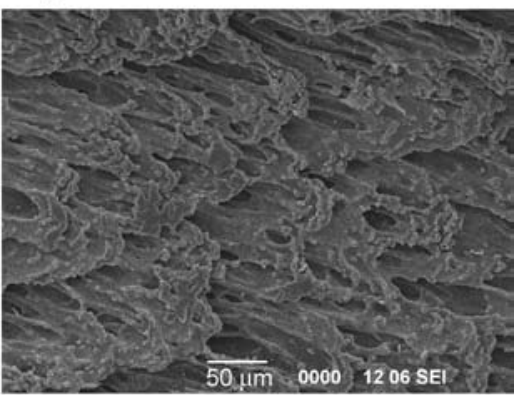

d)

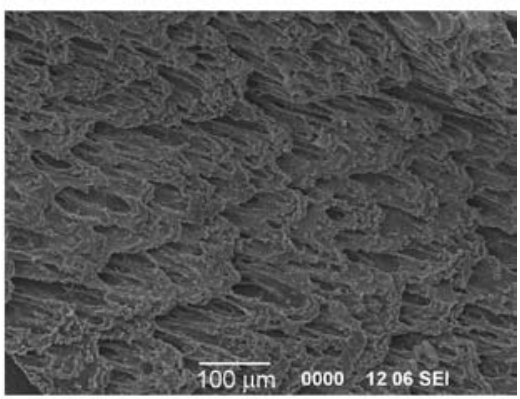

Figure 7. $\mathrm{a}-\mathrm{b})$ Macroscopic observation of PLGA scaffold obtained with $\mathrm{NaCl}$ as pore former and c-f) SEM images of PLGA material obtained in the experiment with sodium chloride as porogen and water as solvent of the porogen

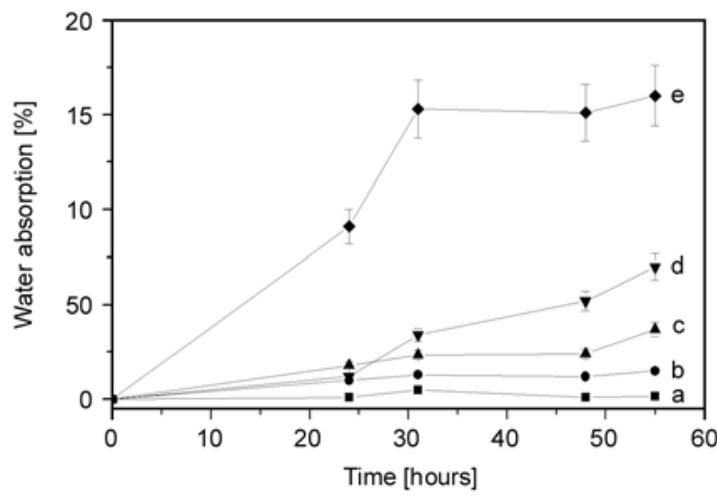

Figure 8. Change of water absorption of various PLGA porous samples in comparison with non-porous PLGA as function of time: a) non-porous PLGA particles; b) porus PLGA material obtained with silicone oil as porogen and $n$-heptan as solvent of the porogen; c) with paraffin as porogen and cyclohexane as solvent of the porogen; d) with hydrogen peroxide as porogen and e) with sodium chloride as porogen and water as solvent of the porogen medium during the 39 days (Table 2). By the end of 39 days, the samples have fully degraded and there were no more traces of them in the solution. Literature describes two approaches for the study of polymeric degradation and they vary according to whether examination is under dynamic or static conditions [45]. In our case degradation of non-porous and porous PLGA was measured under static conditions. It was observed, the degradation medium, in which porous PLGA was suspended, at the beginning of the process of the degradation (measured in the second day), contains much more lactic acid than degradation medium in which PLGA nanoparticles were suspended, which means porous PLGA degrades faster (Table 2 gives cumulative concentrations of the released lactic acid). The releasedelayed phenomenon of macromolecular drug delivery system at the early stage of the degradation was eliminated in the case of porous PLGA 
Table 2. The concentration of lactic acid calculated on the basis of absorbance at the wavelength of absorption maximum at $220 \mathrm{~nm}$ from spectra recorded after 39 days of the degradation and calibration curve for lactic acid

\begin{tabular}{|l|c|c|c|c|}
\hline \multirow{2}{*}{} & \multicolumn{2}{|c|}{ Absorbance at 220 nm } & \multicolumn{2}{c|}{ Concentration [mg/ml] } \\
\cline { 2 - 5 } & After two days & After 39 days & After two days & After 39 days \\
\hline PLGA & 0.068 & 0.507 & 0.07 & 1.00 \\
\hline Porous PLGA ( $\mathrm{NaCl}$ as pore former) & 0.242 & 0.561 & 0.42 & 1.12 \\
\hline
\end{tabular}

The data are presented as mean values of three independent

obtained with sodium chloride as porogen and water as solvent of the porogen. The explanation for this phenomenon is very simple. During the degradation process the non-porous PLGA particles are in a very close contact which brings to higher agglomeration of the particles and creation of the porous film [18]. The degradation of the PLGA becomes faster when the degradation medium passes through the polymeric membrane, i.e. the degradation is more rapid when polymeric film becomes sufficiently porous. We have already elaborated this process elsewhere [18]. The sample obtained in the experiment with sodium chloride as porogen and water as solvent of the porogen has already porous structure so degradation becomes more rapid without delay, i.e. immediately and so the release of, potentially encapsulated, drug will be starting, also, without delay.

\section{7. pH measurements}

The $\mathrm{pH}$ of PBS solution has begun to decrease, approximately, after $48 \mathrm{hrs}$ of storage, in the case of porous PLGA when sodium chloride was used as pore former as well as in the case of non-porous PLGA (Figure 9). This is presumably due to an accumulation of lactic and glycolic acid. PLGA degrades via backbone hydrolysis (bulk erosion) and the degradation products are the monomers, lactic acid and glycolic acid. It could be expected that the faster degradation of the lower molar mass fraction, present in copolymer, increases the local acidity, thereby, accelerating the hydrolysis of higher molar mass species. In another words, when acid accumulation creates a local $\mathrm{pH}$ drop, catalytic degradation of the polymer itself occurs [24]. The pH of PBS medium in which porous PLGA scaffold was stored decreases faster at the beginning of the degradation and the reason for these phenomena is that the degradation medium diffuse through the sample i.e. medium passes into pores of the sample. Also, as already noted, PLGA porous scaffolds obtained with sodium chloride as porogen exhibited

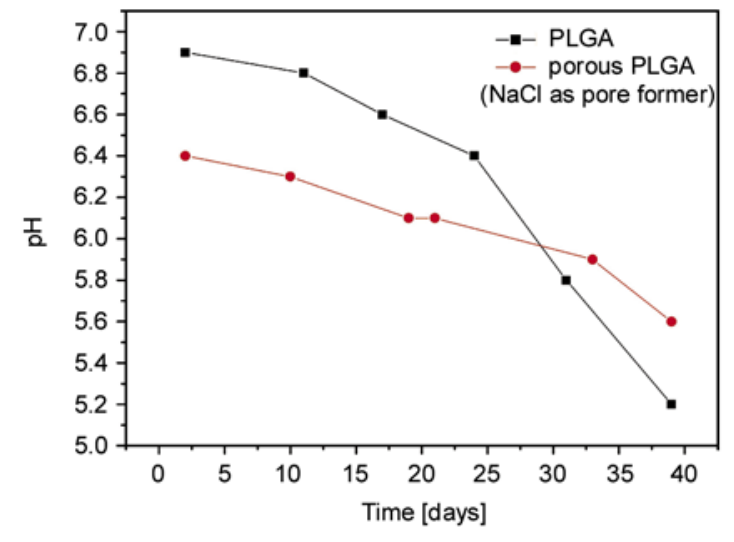

Figure 9. Changes in the $\mathrm{pH}$ of the degradation medium with time for the PLGA in the case of a) non porous PLGA nanoparticles and b) porous PLGA obtained when sodium chloride was used as pore forme

higher water absorption than other samples and degradation medium in which PLGA porous scaffolds were suspended contains more lactic acid after two days of the degradation. In the case of nonporous PLGA, during the degradation process the particles are in a very close contact which leads to higher agglomeration of the particles and formation of the porous film [18]. The degradation becomes more rapid when polymeric film becomes sufficiently porous. Because of that, after approximately 20 days, degradation of newly generated porous film (initially non-porous PLGA particles) becomes faster and thus it comes to a faster decrease of $\mathrm{pH}$ than in the case of $\mathrm{pH}$ of the porous PLGA scaffold. Thin PLGA films degraded faster than thick scaffold because thin films had a greater surface area to volume ratio and thus a greater extent of degradation medium uptake.

\section{Conclusions}

The physicochemical method has produced nonagglomerated PLGA nanospheres with spherical and uniform shapes. We have shown that PLGA nanospheres are not cytotoxic and they do not induce increased production of ROS for HepG2 cells in 
vitro, which is an optimistic result for usage of PLGA nanospheres for in vivo applications. Porous poly (DL-lactid-co-glycolid) materials were fabricated using different porogens, silicon oil, paraffin, hydrogen peroxide or sodium chloride. It was found that silicon oil as porogen has no major impact on the formation of pores in the sample. Paraffin leads to the formation of pores with irregular shapes and without a good interconnectivity. When hydrogen peroxide was used as porogen, sample with high porosity and with good interconnectivity was obtained but pore size has mean value only of about $10 \mu \mathrm{m}$. SEM micrographs showed that porous PLGA scaffolds obtained in the experiment with sodium chloride as porogen and water as solvent of the porogen had apparently uniform pore morphology with spherical pore in shape and well controlled three-dimensional interconnected network. These PLGA samples are highly porous with similar porosity values and exhibit higher water absorption than porous samples obtained with another porogens. To eliminate the release-delayed phenomenon of PLGA macromolecular drug delivery system at the early stage, biodegradable implantable drug delivery system with porous structure was designed when sodium chloride was used as pore former and by physicochemical solvent/non-solvent method. An important clinical application of this porous PLGA material is reflected in the case of many chronic diseases where long term therapy is needed, but one has to start treatment immediately. Besides for the controlled delivery of drugs, this porous material is also very suitable in many other biomedical applications, for example in tissue engineering because it provides the required properties needed for the survival of cells (cell adhesion, proliferation, migration, and/or differentiation) and formation of tissue.

\section{Acknowledgements}

This study was supported by the Ministry of Science and Technological Development of the Republic of Serbia, under Grant No. III45004: Molecular designing of nanoparticles with controlled morphological and physicochemical characteristics and functional materials based on them. The authors would like to thank to Ljiljana Kandić for X ray measurements, Srečo Škapin for FESEM analysis and Slobodan Milonjić for zeta potential measurements.

\section{References}

[1] Lim H. J., Ghim H. D., Choi J. H., Chung H. Y., Lim J. O.: Controlled release of BMP-2 from alginate nanohydrogels enhanced osteogenic differentiation of human bone marrow stromal cells. Macromolecular Research, 18, 787-792 (2010). DOI: $10.1007 / \mathrm{s} 13233-010-0804-6$

[2] Wang Z. H., Wang Z. Y., Sun C. S., Wang C. Y., Jiang T. Y., Wang S. L.: Trimethylated chitosan-conjugated PLGA nanoparticles for the delivery of drugs to the brain. Biomaterials, 31, 908-915 (2010). DOI: 10.1016/j.biomaterials.2009.09.104

[3] Gupta A., Vara D. S., Punshon G., Sales K. M., Winslet M. C., Seifalian A. M.: In vitro small intestinal epithelial cell growth on a nanocomposite polycaprolactone scaffold. Biotechnology and Applied Biochemistry, 54, 221-229 (2009).

DOI: $10.1042 / B A 20090214$

[4] Pattison M. A., Wurster S., Webster T. J., Haberstroh K. M.: Three-dimensional, nano-structured PLGA scaffolds for bladder tissue replacement applications. Biomaterials, 26, 2491-2500 (2005).

DOI: 10.1016/j.biomaterials.2004.07.011

[5] Thapa A., Miller D. C., Webster T. J., Haberstroh K. M.: Nano-structured polymers enhance bladder smooth muscle cell function. Biomaterials, 24, 2915-2926 (2003).

DOI: $10.1016 / \mathrm{S} 0142-9612(03) 00123-6$

[6] Melchels F. P. W., Feijen J., Grijpma D. W.: A poly (D,L-lactide) resin for the preparation of tissue engineering scaffolds by stereolithography. Biomaterials, 30, 3801-3809 (2009).

DOI: 10.1016/j.biomaterials.2009.03.055

[7] Blanco T. M., Mantalaris A., Bismarck A., Panoskaltsis N.: The development of a three-dimensional scaffold for ex vivo biomimicry of human acute myeloid leukaemia. Biomaterials, 31, 2243-2251 (2010).

DOI: 10.1016/j.biomaterials.2009.11.094

[8] Bonartsev A. P., Livshits V. A., Makhina T. A., Myshkina V. L., Bonartseva G. A., Iordanskii A. L.: Controlled release profiles of dipyridamole from biodegradable microspheres on the base of poly(3-hydroxybutyrate). Express Polymer Letters, 1, 797-803 (2007).

DOI: 10.3144/expresspolymlett.2007.110

[9] Sun K., Li Z. H.: Preparations, properties and applications of chitosan based nanofibers fabricated by electrospinning. Express Polymer Letters, 5, 342-361 (2011).

DOI: $10.3144 /$ expresspolymlett.2011.34

[10] Fuchs S., Jiang X., Gotman I., Makarov C., Schmidt H., Gutmanas E. Y., Kirkpatrick C. J.: Influence of polymer content in Ca-deficient hydroxyapatite-polycaprolactone nanocomposites on the formation of microvessel-like structures. Acta Biomaterialia, 6, 3169-3177 (2010).

DOI: $10.1016 /$ j.actbio.2010.02.001 
[11] Park C. H., Hong Y. J., Park K., Han D. K.: Peptidegrafted lactide-based poly(ethylene glycol) porous scaffolds for specific cell adhesion. Macromolecular Research, 18, 526-532 (2010).

DOI: 10.1007/s13233-010-0517-9

[12] Wang Y., Shi X., Ren L., Wang C., Wang D-A.: Porous poly (lactic-co-glycolide) microsphere sintered scaffolds for tissue repair applications. Materials Science and Engineering C, 29, 2502-2507 (2009).

DOI: $10.1016 /$ j.msec.2009.07.018

[13] Liu S-J., Hsueh C-L., Ueng S. W-N., Lin S-S., Chen JK.: Manufacture of solvent-free polylactic-glycolic acid (PLGA) scaffolds for tissue engineering. AsiaPacific Journal of Chemical Engineering, 4, 154-160 (2009).

DOI: $10.1002 /$ apj.187

[14] Stevanović M., Radulović A., Jordović B., Uskoković D.: Poly(DL-lactide-co-glycolide) nanospheres for the sustained release of folic acid. Journal of Biomedical Nanotechnology, 4, 349-358 (2008).

DOI: $10.1166 / \mathrm{jbn} .2008 .321$

[15] Zhang J., Rana S., Srivastava R. S., Misra R. D. K.: On the chemical synthesis and drug delivery response of folate receptor-activated, polyethylene glycol-functionalized magnetite nanoparticles. Acta Biomaterialia, 4, 40-48 (2008).

DOI: $10.1016 /$ j.actbio.2007.06.006

[16] Abdelwahed W., Degobert G., Stainmesse S., Fessi H.: Freeze-drying of nanoparticles: Formulation, process and storage considerations. Advanced Drug Delivery Reviews, 58, 1688-1713 (2006).

DOI: $10.1016 /$ j.addr.2006.09.017

[17] Zhang H., Chen Z.: Fabrication and characterization of electrospun PLGA/MWNTs/hydroxyapatite biocomposite scaffolds for bone tissue engineering. Journal of Bioactive and Compatible Polymers, 25, 241-258 (2010).

DOI: $10.1177 / 0883911509359486$

[18] Stevanović M., Maksin T., Petković J., Filipič M., Uskoković D.: An innovative, quick and convenient labeling method for the investigation of pharmacological behavior and the metabolism of poly(DL-lactide-coglycolide) nanospheres. Nanotechnology, 20, 335102/1335102/12 (2009).

DOI: $10.1088 / 0957-4484 / 20 / 33 / 335102$

[19] Wei G., Ma P. X.: Polymer/ceramic composite scaffolds for bone tissue engineering. in 'Scaffolding in tissue engineering' (eds.: Ma P. X., Elisseeff J.) CRC Press, Boca Raton, 241-251 (2006).

[20] Sha'ban M., Kim S. H., Idrus R. B. H., Khang G.: Fibrin and poly(lactic-co-glycolic acid) hybrid scaffold promotes early chondrogenesis of articular chondrocytes: An in vitro study. Journal of Orthopaedic Surgery and Research, 3, 17/1-17/10 (2008).

DOI: $10.1186 / 1749-799 X-3-17$
[21] Lee J. J., Lee S-G., Park J. C., Yang Y. I., Kim J. K.: Investigation on biodegradable PLGA scaffold with various pore size structure for skin tissue engineering. Current Applied Physics, 7, 37-40 (2007).

DOI: $10.1016 /$ j.cap.2006.11.011

[22] Dawes G. J. S., Fratila-Apachitei L. E., Mulia K., Apachitei I., Witkamp G-J., Duszczyk J.: Size effect of PLGA spheres on drug loading efficiency and release profiles. Journal of Materials Science: Materials in Medicine, 20, 1089-1094 (2009).

DOI: $10.1007 / \mathrm{s} 10856-008-3666-0$

[23] Blanco M. D., Sastre R. L., Teijón C., Olmo R., Teijón J. M.: Degradation behaviour of microspheres prepared by spray-drying poly(D,L-lactide) and poly(D,Llactide-co-glycolide) polymers. International Journal of Pharmaceutics, 326, 139-147 (2006).

DOI: 10.1016/j.ijpharm.2006.07.030

[24] Ito F., Fujimori H., Makino K.: Factors affecting the loading efficiency of water-soluble drugs in PLGA microspheres. Colloids and Surfaces B: Biointerfaces, 61, 25-29 (2008).

DOI: $10.1016 /$ j.colsurfb.2007.06.029

[25] Krebs M. D., Sutter K. A., Lin A. S. P., Guldberg R. E., Alsberg E.: Injectable poly(lactic-co-glycolic) acid scaffolds with in situ pore formation for tissue engineering. Acta Biomaterialia, 5, 2847-2859 (2009). DOI: $10.1016 /$ j.actbio.2009.04.035

[26] Stevanović M., Uskoković D.: Poly(lactide-co-glycolide)-based micro and nanoparticles for the controlled drug delivery of vitamins, review article. Current Nanoscience, 5, 1-14 (2009).

[27] Rabelo D., Lima E. C. D., Reis A. C., Nunes W. C., Novak M. A., Garg V. K., Oliveira A. C., Morais P. C.: Preparation of magnetite nanoparticles in mesoporous copolymer template. Nano Letters, 1, 105-108 (2001). DOI: $10.1021 / \mathrm{n} 1005533 \mathrm{k}$

[28] Kim S., Choi J. E., Choi J., Chung K-H., Park K., Yi J., Ryu D-Y.: Oxidative stress-dependent toxicity of silver nanoparticles in human hepatoma cells. Toxicology in Vitro, 23, 1076-1084 (2009).

DOI: $10.1016 /$ j.tiv.2009.06.001

[29] Schins R. P. F., Knaapen A. M.: Genotoxicity of poorly soluble particles. Inhalation Toxicology, 19, 189-198 (2007).

DOI: $10.1080 / 08958370701496202$

[30] Osseni R. A., Debbasch C., Christen M-O., Rat P., Warnet J-M.: Tacrine-induced reactive oxygen species in a human liver cell line: The role of anethole dithiolethione as a scavenger. Toxicology in Vitro, 13, 683688 (1999).

DOI: $10.1016 / \mathrm{S} 0887-2333(99) 00050-8$

[31] Stevanović M., Savić J., Jordović B., Uskoković D.: Fabrication, in vitro degradation and the release behaviours of poly(DL-lactide-co-glycolide) nanospheres containing ascorbic acid. Colloids and Surfaces B: Biointerfaces, 59, 215-223 (2007). DOI: $10.1016 /$ j.colsurfb.2007.05.011 
[32] Petković J., Žegura B., Stevanović M., Drnovšek N., Uskoković D., Novak S., Filipič M.: DNA damage and alterations in expression of DNA damage responsive genes induced by $\mathrm{TiO}_{2}$ nanoparticles in human hepatoma HepG2 cells. Nanotoxicology, in press (2011). DOI: $10.3109 / 17435390.2010 .507316$

[33] LeBel C. P., Ischiropoulos H., Bondy S. C.: Evaluation of the probe $2^{\prime}, 7^{\prime}$-dichlorofluorescin as an indicator of reactive oxygen species formation and oxidative stress. Chemical Research in Toxicology, 5, 227-231 (1992). DOI: $10.1021 / \mathrm{tx} 00026 \mathrm{a} 012$

[34] Zhang J., Wu L., Jing D., Ding J.: A comparative study of porous scaffolds with cubic and spherical macropores. Polymer, 46, 4979-4985 (2005).

DOI: $10.1016 /$ j.polymer.2005.02.120

[35] Cai Q., Yang J., Bei J., Wang S.: A novel porous cells scaffold made of polylactide-dextran blend by combining phase-separation and particle-leaching techniques. Biomaterials, 23, 4483-4492 (2002). DOI: 10.1016/S0142-9612(02)00168-0

[36] Perugini P., Genta I., Conti B., Modena T., Pavanetto F.: Periodontal delivery of ipriflavone: New chitosan/ PLGA film delivery system for a lipophilic drug. International Journal of Pharmaceutics, 252, 1-9 (2003). DOI: 10.1016/S0378-5173(02)00602-6

[37] Boccaccini A. R., Blaker J. J., Maquet V., Chung W., Jérôme R., Nazhat S. N.: Poly(D,L-lactide) (PDLLA) foams with $\mathrm{TiO}_{2}$ nanoparticles and PDLLA/TiO - -Bioglass $^{\circledR}$ foam composites for tissue engineering scaffolds. Journal of Materials Science, 41, 3999-4008 (2006). DOI: $10.1007 / \mathrm{s} 10853-006-7575-7$

[38] Woo Y. I., Lee M. H., Kim H-L., Park J-C., Han D-W., Kim J. K., Tsubaki K., Chung K-H., Hyun S. O., Yang Y-I.: Cellular responses and behaviors of adiposederived stem cells onto $\beta$-glucan and PLGA composites surface-modified by microwave-induced argon plasma. Macromolecular Research, 18, 90-93 (2010). DOI: $10.1007 / \mathrm{s} 13233-009-0125-9$
[39] Kiremitçi-Gümüşderelioğlu M., Deniz G.: Synthesis, characterization and in vitro degradation of poly(DLlactide)/poly(DL-lactide-co-glycolide) films. Turkish Journal of Chemistry, 23, 153-162 (1999).

[40] Yang T-H., Dong A., Meyer J., Johnson O., Cleland J., Carpenter J.: Use of infrared spectroscopy to assess secondary structure of human growth hormone within biodegradable microspheres. Journal of Pharmaceutical Sciences, 88, 161-165 (2000).

DOI: $10.1021 /$ js $980423 n$

[41] Arrieta A., Mera S., Diamant R., Fernández-Guasti M., Sosa R., Escobar-Alarcón L., Muñoz A. F., Haro-Poniatowski E.: Synthesis and characterization of sodium chloride thin films obtained by pulsed laser deposition. Applied Physics A: Materials Science and Processing, 69, S491-S493 (1999).

DOI: $10.1007 / \mathrm{s} 003399900321$

[42] Belu A., Mahoney C., Wormuth K.: Chemical imaging of drug eluting coatings: Combining surface analysis and confocal Raman microscopy. Journal of Controlled Release, 126, 111-121 (2008).

DOI: $10.1016 /$ j.jconrel.2007.11.015

[43] Stevanović M., Ignjatović N., Jordović B., Uskoković D.: Stereological analysis of the poly-(DL-lactide-coglycolide) submicron sphere prepared by solvent/nonsolvent chemical methods and centrifugal processing. Journal of Materials Science: Materials in Medicine, 18, 1339-1344 (2007). DOI: $10.1007 / \mathrm{s} 10856-007-0156-8$

[44] Chen H., Ding Y., Tan C.: Rheological behaviour of nanofluids. New Journal of Physics, 9, 367/1-367/23 (2007).

DOI: $10.1088 / 1367-2630 / 9 / 10 / 367$

[45] Pukánszky B., Nagy T. T., Kelen T., Tüdős F.: Comparison of dynamic and static degradation of poly(vinyl chloride). Journal of Applied Polymer Science, 27, 2615-2623 (1982).

DOI: $10.1002 /$ app.1982.070270730 